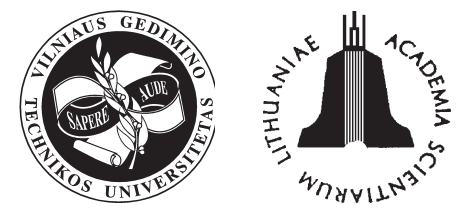

ISSN 1648-4142 TRANSPORT

http:/www.vtu.lt/english/editions

TRANSPORT - 2005, Vol XX, No 2, 62-65

\title{
PARAMETERS OF FUEL JET EXTRACTION IN VIDEO
}

\author{
Alexsander Eskov' ${ }^{1}$, Andrey Svistula ${ }^{2}$, Alexey Dolmatov \\ Altai State Technical University, 46 Lenin St., 656099 Barnaul, Russia. \\ Telffax: (+3852260516), Tel: (+3852368475). \\ Received 2004-11-10; accepted 2005-01-25
}

\begin{abstract}
The statistical rating of the image of a fuel jet allows to allocate the zones with homogeneous brightness, to define the distribution of masses and the concentration in a fuel jet and to look after their dynamics. In the research the coding with the usage of Byeses's definition of the aposteriory probability of the presence of the given brightness in the image is described. The bright zones of a fuel jet in a diesel engine will give to the conclusion how the fuel is distributed in the engine combustion camera.
\end{abstract}

Keywords: recording and image processing, CCD, picture system, fuel jet.

\section{Introduction}

The ecological and economical requirements for modern internal combustion engines influence the exploring of methods and the creation of devices to control the quality of fuel dispersion in the engine combustion camera. The process of fuel jet extraction, namely the equal fuel volume dispersion, determines the engine characteristics. It is important to solve the problem of mass distribution and fuel concentration in the combustion camera and changing these parameters within each jet extraction [1].

The formation of the dispersion flow of the particles of the condensed phase predetermines the exploring of effect uniform digital technologies of processing results received by optical uncontact diagnostics of disperse flows in real time on the basis of integrated fotodiodes structures (matrix or linear) and microprocessor systems of registration and management. The use of CMOS-fotodiodes gauges together with stroboscopic illumination of fuel jets allows to determine the mass transfer distribution of fuel in a body of a cold fuel flow, to register borders of a flow, a corner of a jet cone in the mode of real time. The consecutive shooting of several staffs of the same frame fuel jet gives a picture of speeds of moving various masses for time intervals between the moments of registration. Besides the registration by stroboscopic illumination gives the information about the degree of absorption of a falling light flow by a fuel

\footnotetext{
${ }^{1}$ alesc@newmail.ru

2sae59@mail.ru
}

jet, or about the areas of single light dispersion for the further definition of dispersion fuel composition in these areas using the small corners method [2].

During the experiments it was established that the jets, flowing out from the nozzle holes, pulse and the character of pulsation depends on the geometry of the nozzle, physical abilities of a fluid, gas medium and their relative speed. Under some circumstances these pulsations get into decay. Thus the factors influencing on the length of a solid part (up to decay) of a jet are determined experimentally. The bigger the diameter of a nozzle hole, the longer a solid part. The expiration speed influences on the length of a nondecayed part of a jet and this dependence is maximum.

During the process of dispersion the fluid jet decays in an uncountable amount of drops of different sizes. Modern scientists consider that the formation of a fuel jet represents a casual process. It means that having registered all parameters of injection it's impossible to get the drops of the same size and identical speed in the same place of the jet. The characteristic feature of a fuel jet of modern diesel engines is the alternating pressure of fuel injection.

Modern matrix and linear photosensors together with stroboscopic illumination in CMOS structure or exterior stroboscopic source of light radiation allow to register the integrated process of fuel jet at a little period of time (about $200 \mathrm{~ms}$ ) with the purpose of its further registration on the computer. The main characteristics describing fuel jet dispersion are the corner of a jet cone, the length of a fuel jet, the availability of optical dissimiliarities and the distribution of 
fuel in a body of a jet. Devices of images registration on the basis of integrated photodiodes matrix structures and CCD structures with the use of digital systems allow to conduct optical registration in the mode of real time and the diagnostics of disperse flow that is a fuel jet.

\section{The test bench for registration and processing of fuel jet images}

The system of images entrance VS-CCT-285-2001 "Videoscan" is used for the investigation of the structure of optical dissimilarities in fuel jet dispersion which defines the character of fuel jet extraction. The system works on the basis of matrix ICX285AL by SONY, the picture size element $1360 * 1024$ and allows to conduct separate phases description of fuel jet development by several form injections. The complex of model conceptions on the morphology of elements structure is on the basis of automatic analysis.

The chair of engine-building of Altai State Technical University with the chair of experimental physics created the bench for registration and processing of fuel jet images. The bench includes: diagnostic camera (1) representing a tube with diameter $1 \mathrm{~m}$, with spherical bottom and spherical cover (Fig 1). The valve (3) of stroboscopic illumination fuel jet, injected by the nozzle is built at the bottom of camera nozzle. At the top of camera cover there is head CCD (5) with optical registered system. Fuel flows into the nozzle by pipeline from fuel pump (6) being on special bench of testing and processing fuel pumps (7) "MOTOR-PAL". Fuel bench "MOTOR-PAL" includes an electric motor (8) which rotates camshaft fuel pump (9). Fly-wheel (10) with the turnover angle is on the camshaft.

The bench structure "MOTOR-PAL" includes a timer which is used for time registration from the beginning of the injections made by the operator. The impulse of stroposcope flash and synchronization of

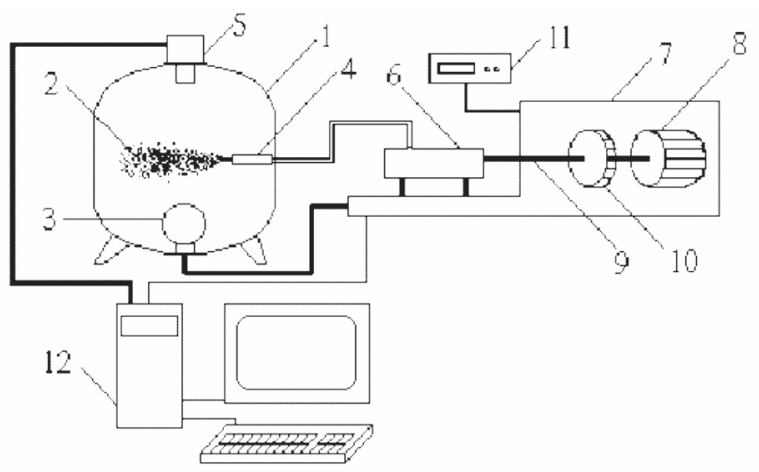

Fig 1. Scheme of the experimental bench for registration and processing of fuel jet images picture size entrance system VS-CTT-2852001"Videoscan" (12) is at the end. On arrival from the bench "MOTOR-PAL" the impulse picture-size entrance system VS-CTT-285-2001 "Videoscan" memorized staff in accordance with the established earlier exposure time. Thus, "Videoscan" settles the fuel jet image during illumination.

\section{Analysing of the results of fuel jet images}

A special programme allows to process the data of fuel jet image (to measure the corner of the jet (Fig 2), the length of fuel flow, to contrast optical dissimiliarities and receiption of legible borders), to get "strong copy" of the image on the printer and also to create data bank information.

The corner of the jet is determined by the difference of pitch corners of two straight lines with known coordinates of 2 points on each straight line.

The point coordinates are determined by the operator or automatically. The image contrastation occurs at processing by gradient-courses mask. Processed image results have a brightly expressed contrast in places of gradient direction of initial image which coincides with the direction of mask action (Fig 3).

The fuel jet length is determined by the comparison of fuel jet borders and the measured object with known sizes. The optical system of a diagnostic camera follows this object. The largest error of length definition of a fuel jet makes the size of the mesh CCD multiplied on the scale of the magnification of an optical system. The mesh CCD measures $6,45 \times 6,45$ microns. Different methodics and numerical algorithms of definition of the main integrated parameters

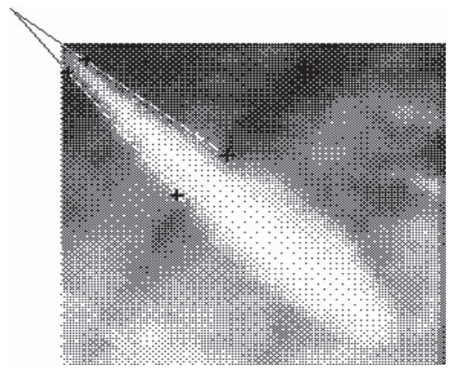

Fig 2. To measure the corner of the jet (the corner is $9,3^{\circ}$ )

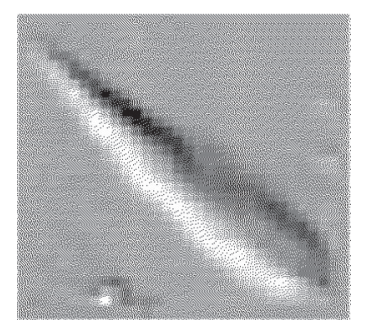

Fig 3. The fuel jet imaging which coincides with the direction of mask action is "those-west" 
of a dispersion process have passed the approbation on the basis of digital data images of a fuel jet: the selection of optical dissimilarities and inspissation of fuel cloud, their middle sizes and statistical characteristics, the corners of fuel jet, effective area and nonsymmetry.

Characteristic bright zones in the fuel jet image received with the help of a specialised digital television system can be distinguished to rate the mass transfer parameters in them. Each such zone has appropriate optical density which is proportional to the concentration of particles in it.

The deforming factors which are brought by a registration system to the receiving data should be taken into account before implementing the analysis of the video object identification of a fuel jet. These factors have the determined character as the function of point dispersion of a registration system and casual noise. However, if their impact is not too large, the statistical parameters of the image will not be changed essentially.

The authors offer a coding method of the fuel jet image statistically the most probable brightness for the suppression of deforming factors and highlighting the characteristic bright zones. The coding is made with the use of Byese's definition of aposteriory probability of the presence of the given brightness in the image. The iterative algorithm based on this method modifies the histogram of brightness of the initial image so that it would contain statistically the most probable brightness. During this operation the probability of a mistake of the given transformation named as a risk is calculated. The algorithm finishes the work

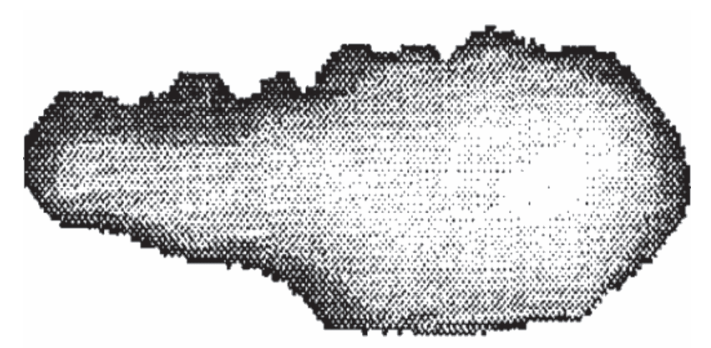

Fig 4. Initial image of a fuel jet

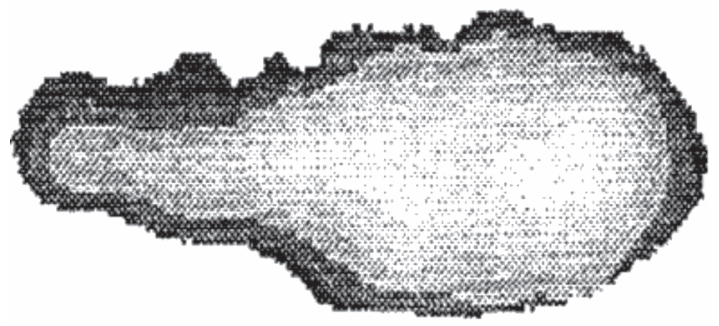

Fig 5. Statistical rating when the risk reaches a beforehand given level. Thus the rating of the image of a fuel jet with the given probability of a mistake is done [3, 4]. In Figure 4 the initial image of a fuel jet and in Figure 5 its statistical rating are shown.

In Figures 6, 7 the histogram of brightness of the initial and processed image of a fuel jet representing the dependence of the quantity of pixels of the image from brightness is shown.

Analyzing the histogram of brightness of the processed image, we count up the quantity of pixels in a fuel jet object appropriate to every bright zone. Proceeding from the quantity of pixels of a jet, we build percentage ratio of the areas of bright zones. If we consider a fuel jet as a rotation body we can establish the mass of particles in each zone with characteristic brightness and also owing to the areas or volumes of the zones we can estimate their concentration knowing the mass of an injected doze of fuel.

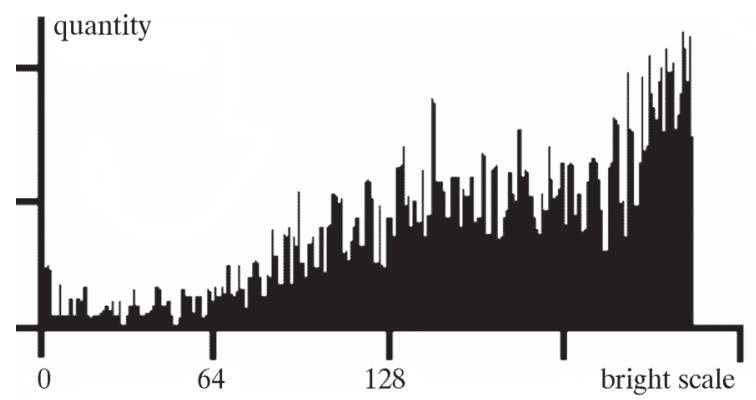

Fig 6. Histogram of brightness of the initial image

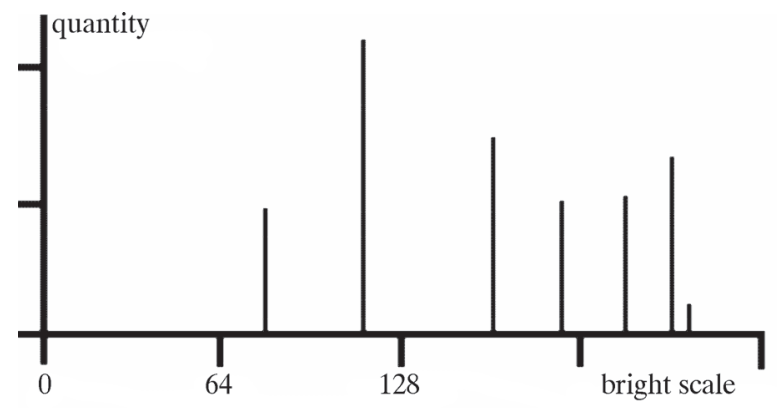

Fig 7. Histogram of brightness of the processed image

\section{Conclusion}

Thus, the statistical rating of the image of a fuel jet allows to allocate the zones with homogeneous brightness, to define the distribution of masses and the concentration in a fuel jet and to look after their dynamics. 
In conclusion the possibility of the usage of the experimental bench for registration and processing of a fuel jet images under conditions of real-time manufacturing the fuel sprayers with the purpose of fuel jet characteristics control and further sprayers selection for similar groups can be marked.

\section{References}

1. Sviridov, Yu. B. Generator of Fuel Jet and Combustion in Diesel Engines (Смесеобразование и сгорание в дизелях). Leningrad: Machineindustry, 1972. 224 p. (in Russian).

2. Mie, G. Beitrage zur Optic truber Meiden, speziell kolloidaler Metallosunden. Annalen der Phisik, Vol XXV, No 2, Berlin, 1908, 377 p. (in German).

3. Duda, R.; Hart, P. Pattern Recognition and Analysis of Stages (Распознавание образов и анализ сцен). Moscow, 1976. 511 p. (in Russian).

4. Eskov, A. V.; Dolmatov, A. V. Statistical Rating of Mass Transfer Parameters of Fuel Jets. Vestnik of the Altai State Technical University, No 2, 1999, p. 77-78. 